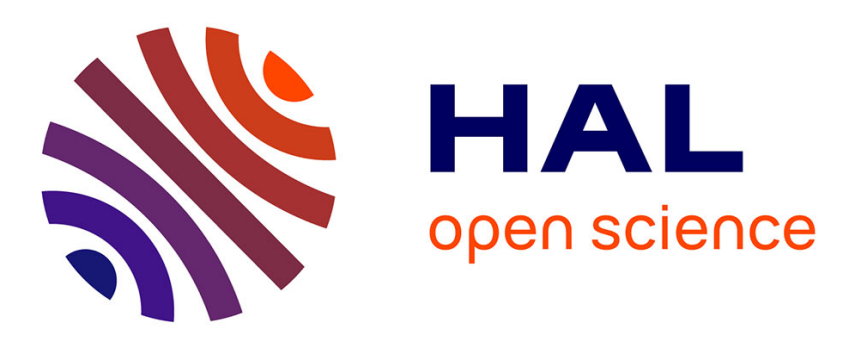

\title{
Similarity Based Cross-Section Segmentation in Rough Log End Images
}

\author{
Rudolf Schraml, Andreas Uhl
}

\section{To cite this version:}

Rudolf Schraml, Andreas Uhl. Similarity Based Cross-Section Segmentation in Rough Log End Images. 10th IFIP International Conference on Artificial Intelligence Applications and Innovations (AIAI), Sep 2014, Rhodes, Greece. pp.614-623, 10.1007/978-3-662-44654-6_61 . hal-01391368

\section{HAL Id: hal-01391368 https://hal.inria.fr/hal-01391368}

Submitted on 3 Nov 2016

HAL is a multi-disciplinary open access archive for the deposit and dissemination of scientific research documents, whether they are published or not. The documents may come from teaching and research institutions in France or abroad, or from public or private research centers.
L'archive ouverte pluridisciplinaire HAL, est destinée au dépôt et à la diffusion de documents scientifiques de niveau recherche, publiés ou non, émanant des établissements d'enseignement et de recherche français ou étrangers, des laboratoires publics ou privés.

\section{(c)(1)}

Distributed under a Creative Commons Attribution| 4.0 International License 


\title{
Similarity based cross-section segmentation in rough log end images
}

\author{
Rudolf Schraml and Andreas Uhl \\ University of Salzburg, Jakob Haringer Str. 2, \\ 5020 Salzburg, Austria
}

\begin{abstract}
This work treats cross-section (CS) segmentation in digital images of rough wood log ends. Existing CS segmentation approaches are focused on computed tomography CS images of logs and no approach and experimental evaluation for digital images has been presented so far. Segmentation of cross-sections in rough log end images is a prerequisite for the development of novel log end analysis applications (e.g. biometric $\log$ recognition or automated log grading). We propose a simple and fast computable similarity-based region growing algorithm for CS segmentation. In our experiments we evaluate different texture features (Local binary patterns \& Intensity histograms) and histogram distances. Results show that the algorithm achieves the most accurate results in combination with intensity histograms and the earth movers distance. Generally, we conclude that for certain applications simple texture features and a matured distance metric can outperform higher-order texture features and basic distance metrics.
\end{abstract}

Keywords: Wood imaging, Cross-section analysis, Cross-section segmentation, Rough log end images

\section{Introduction}

In case of digital images of log ends there exist just a few publications which deal with cross-section (CS) analysis. The works of [1, [2, [3, ,4, [5] present approaches for pith estimation and in [2], [6], [7, ,8 annual ring analysis approaches are proposed. Present CS analysis literature focuses on computed tomography (CT) cross-section images. Compared to conventional log end images, CT crosssection images do not exhibit disturbances due to cutting or dirt. Furthermore, the wood properties are clearly visible and distinguishable. This enables segmentation of the entire CS and of wood properties/ defects using basic thresholding techniques (literature review see [9]). To our knowledge, no publications related to cross-section segmentation in digital images of rough log ends were published. Digital images of rough log ends can be taken at little cost and at almost every stage in the wood processing chain. The location, size and shape of the CS are crucial to perform sophisticated image analysis tasks. Thus, segmentation is a prerequisite for the development of novel applications (e.g. automated log grading or biometric traceability of logs - see [10]) using images of rough log 
end faces. Beneath a high segmentation accuracy, the timing performance of any segmentation approach is crucial for any real world application.

Basically, a cross-section is built up of an annual ring texture which varies strongly locally. Variations are caused by the the circular alignment, different widths, colour features and disturbances of annual rings. Hence, CS segmentation is a typical task for region-based segmentation approaches which are able to segment textured regions. State-of-the-art region-based segmentation approaches are based on graph cuts $([11,[12])$, active contours ([13, [14]) or mean shift ([15]) algorithms. In the past two decades manifold publications showed up high level segmentation algorithms combined with well matured texture features like Markov-random-field statistics ([16]), Wavelet features ([17],[18]), Gabor filters ([19]) and Fractal features ([20]). Furthermore, there exist many more textural spectral-temporal features (literature review see [21]) and various new texture features and extensions were published (e.g. [22], [23], 24], 25]).

Recently, in [26, 27, the region-based active contour approach proposed by 14 is extended to deal with similarity of adjacent patches which enables segmentation of inhomogeneous textured regions. This approach is applicable to CS segmentation, but requires a high computational effort. For this first work dealing with CS segmentation we pay attention to the timing performance. Instead of using active contours we utilize an ease and fast computable region-growing approach which is based on similarity of adjacent patches. Similarity is assessed by comparing the histogram distances of texture features. For this purpose two fast computable features are evaluated as texture features: Intensity- and local binary pattern (LBP) histograms. The utilized region-growing approach can be combined with any comparable texture feature.

This work contributes to the development of new CS analysis applications suggesting a simple, accurate and fast computable approach for CS segmentation in rough log end images. A testset consisting of 108 different rough log end images is utilized to perform experiments. Results show that for the utilized algorithm simple intensity histograms as texture features and the earth movers distance (EMD) outperform a well-matured texture descriptor - the LBP operator. Additionally, the experiments examine different settings and parameters required for the segmentation algorithm.

At first, Section 2 introduces the utilized texture analysis methods. In Section 3 the region-growing based CS segmentation algorithm is outlined. The experimental setup and results are presented in Section 4 followed by the conclusion in Section 5 .

\section{Texture analysis background}

This section summarizes two different texture features and a set of histogram distances utilized for the CS segmentation algorithm and the experiments.

Intensity Histograms do not extract information about the topology of the pixels. Generally, histograms of images or image sections are probability distributions describing the frequency of each color or gray value. 
Local Binary Patterns (LBPs) were introduced by [28. In counting the frequency of micro texels in an image or image section, LBPs unify statistical and structural principles of texture analysis. For each pixel in an image or image section the local binary pattern is computed by analysing $\mathrm{N}$ neighbours in a circular neighbourhood. The occurrence of each of the $2^{N}$ possible patterns is stored into a feature histogram, where each bin represents a single pattern and its frequency. Additionally to the generic formulation of the LBP operator, the authors of [29] and [30] introduced uniform, rotation invariant and multi-scale LBP variations.

Histogram Distances are used as similarity measures between feature histograms of adjacent image sections. Four bin-by-bin distances (L1-Norm - $L_{1}$, L2-Norm - $L_{2}$, Chi-quadrat distance - $X^{2}$, Hellinger distance - $H$ ) and one crossbin distance (EMD) are examined. The EMD [31] computes the minimal cost required to transform one histogram $(\mathrm{P})$ into another $(\mathrm{Q})$. In case of two onedimensional probability distribution functions $\mathrm{P}$ and $\mathrm{Q}$ the EMD is simply given by the $L_{1}$ norm between their cumulative distribution functions.

\section{Cross-section segmentation algorithm}

Our algorithm is inspired by the EMD-region-based level set formulation in [14, 27, and is adopted to a region growing procedure. The procedure is based on similarity of adjacent image sections. For this purpose, the image is subdivided into blocks. The block size and the overlapping factor between the blocks are crucial parameters which strongly influence the segmentation accuracy and timing performance. Our algorithm is subdivided into three consecutive stages which are described subsequently.

\subsection{Cluster initialisation}

For the selection of seed blocks and the subsequent initialisation of clusters the position of the pith is utilized. We utilize the suggested algorithm and configuration presented in [5]. This pith estimation algorithm computes local orientations in the Fourier Spectra of image sections. The pith position is determined by intersection of the local orientations.

A predefined number of blocks (e.g. 4) which are equally distributed close around the pith position are selected. Subsequently, for each single seed block the adjacent neighbours in a four-neighbourhood are added and then initialised as a single cluster.

Each cluster is initialised by computing three features which describe the contained texture: mean gray value/variance, mean entropy/variance and mean intensity histogram or LBP histogram distances between the blocks of the cluster and the corresponding variance. 


\subsection{Growing procedure}

The cluster growing procedure starts with the selection of one of the initialised clusters. Then the four-neighbourhood of each border block is analysed. Only neighbours which are not allocated to a cluster are used as candidate blocks.Each candidate block is compared to the cluster which leads to a decision whether or not the block is allocated to the cluster.

In Fig. 1 the blocks of a cluster and the decision making procedure are illustrated. For the block labelled B3 three candidate blocks $(\mathrm{nb} 1, \mathrm{nb} 2, \mathrm{nb} 3)$ are available. The final decision whether one of the candidates (e.g. nb2) is allocated requires that each of the three comparisons satisfies predefined conditions. For example, the mean gray value of the block must range between the cluster mean gray value $+/$ its variance. The feature-variances are used to regulate the restrictiveness of the decision making procedure. For this purpose, the variance is multiplied by a factor ranging from $[-2,2]$. Preliminary tests showed that other factors are either too restrictive or too tolerant for the growing procedure. If the block is allocated to the cluster, the cluster features are updated. The procedure continues with the next border block until no border block has further candi-

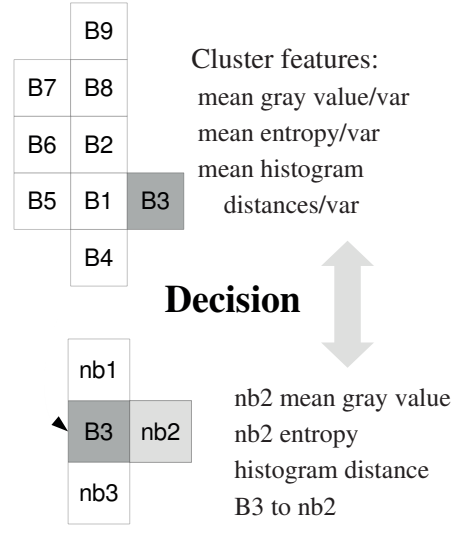

Fig. 1. Illustration of the cluster growing procedure date blocks that fit to the cluster. Then the algorithm continues with the next cluster. The cluster-growing algorithm finishes after all clusters are processed. Finally, the clusters are merged and it is assumed that the merged cluster represents the area of the CS.

An exemplary result is shown in Fig. 2. It is clearly visible that the resulting cluster covers the entire CS. Unfortunately, similar textured CSs in the background are difficult to distinguish from the main CS. This problem is visible in the bottom right of Fig. 2 .

In three consecutive steps the final estimate of the CS boundary is computed. First, all cluster blocks which have at least one neighbour that is not in the cluster are selected (see Fig. 3a). This step reduces the amount of blocks and all of the blocks representing parts of the CS boundary still remain. In the second step, it is assumed that CSs are ap-

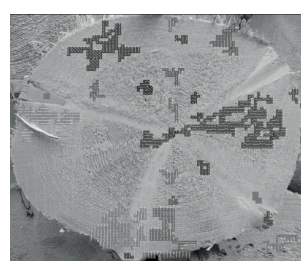
proximately elliptical. First, a circle is fitted into the point cloud of the remaining blocks. Blocks within the circle are removed. This improves the accuracy of the ellipse fitting procedure. After ellipse fitting 
all blocks located outside of the ellipse are removed (see Fig. 3b). In the best case and especially for elliptical CSs this procedure decreases the probability of including incorrectly segmented blocks (e.g. caused by neighboured CSs in the background).

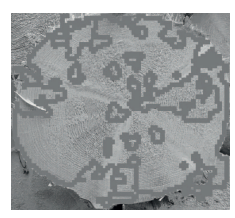

(a) Select border blocks

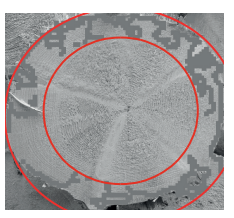

(b) Fit circle/ ellipse

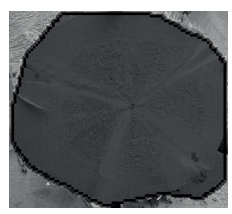

(c) Alpha shape $(\alpha=50)$

Fig. 3. Cross-section (CS) boundary estimation

Finally, the alpha shape [32] of all remaining blocks is computed. Alpha shapes are an approach to compute concave hulls and are a generalisation of convex hulls. They can be tuned by the $\alpha$ parameter. The higher the $\alpha$ value is, the better the alpha shape approximates the convex hull and details of the boundary are neglected. The lower the value of $\alpha$ is, the more details are considered. In contrast, a lower value makes the alpha shape computation vulnerable to large gaps between the outermost blocks. Finally, the alpha shape (see Fig. 3c) is used as a final estimate of the CS boundary.

\section{Experiments}

Three experiments with different configurations are used to assess the general performance as well as the impact of: Different block sizes and half-overlapping/ non-overlapping blocks, using intensity or LBP histograms as texture features, different local histogram distances and different variance factors $\left(V_{f}\right)$ and the circle/ ellipse fitting step. Additionally, one experiment evaluates the timing performance. Testset: 108 (1024x768) circular saw-cut spruce log ends were captured

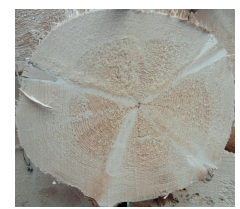

(a) Original image

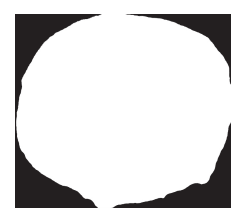

(b) Ground truth mask

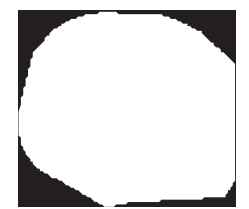

(c) Computed CS mask

Fig. 4. Segmentation accuracy computation masks

with a digital camera (Samsung WB2000), without flash and at approximately the same distance from the log end surface to the camera. The images were converted to grayscale using equal weighting factors. The accuracy for a certain configuration is evaluated computing the mean segmentation accuracy (Mean) 
and the deviation (StDev) across all of the 108 images. $R$ describes the span between the minimum and the maximum of the computed segmentation accuracies. Additionally, the computation time in milliseconds [ms] provides information on the timing performance. The percentage of difference between the pixels of the manually determined ground-truth mask (Fig. 4b) and the computed CS mask (Fig. 4c is defined as the segmentation accuracy.

All steps of the CS segmentation algorithm have been implemented in Java and all experiments have been performed on an Intel Core i7-2620M processor with 2.7 GHZ and 8 GB RAM, JRE 1.7.

\subsection{Experiment \#1 - Intensity histograms}

First, the applicability of intensity histograms as texture features for our algorithm is evaluated. Furthermore, block sizes of $16 \times 16$ and $32 \times 32$, half- and non-overlapping and all histogram distances noticed in Section 2 are examined.

For the cluster growing procedure each histogram distance is tested with variance factors $\left(V_{f}\right)$ in a range between [-2.0,2.0]. For the entropy and mean gray value comparisons a constant $V_{f}=1$ is used. The circle/ellipse fit step is not performed. The best results for all tested configurations are presented in Table 1. For three of four configurations the EMD achieves the best segmentation accuracy. The most accurate results are reached with EMD and 16x16 halfoverlapping pixels blocks (Mean: 5.55, StDev: 3.3). It can be stated that the EMD is a robust and appropriate histogram distance for all tested configurations.

\begin{tabular}{|c|c|c|c|c|c|}
\hline \multicolumn{6}{|c|}{ Blocksize 16x16 } \\
\hline$H_{d}$ & $V_{f}$ & Mean & StDev & $\mathrm{R}$ & {$[\mathrm{ms}]$} \\
\hline \hline \multicolumn{6}{|c|}{ non-overlapping } \\
\hline$L_{1}$ & 0.9 & 7.29 & 3.74 & 17.0 & 312 \\
$L_{2}$ & 1.0 & 7.97 & 3.4 & 15.0 & 310 \\
$\mathrm{H}$ & 0.9 & 7.72 & 4.16 & 17.0 & 312 \\
$X^{2}$ & 0.6 & 8.55 & 4.27 & 17.0 & 310 \\
EMD & $\mathbf{0 . 5}$ & $\mathbf{6 . 4 5}$ & $\mathbf{3 . 4 1}$ & $\mathbf{1 7 . 0}$ & $\mathbf{3 0 4}$ \\
\hline \hline \multicolumn{6}{|c|}{ half-overlapping } \\
\hline$L_{1}$ & -0.5 & 10.04 & 5.64 & 20.0 & 2135 \\
$L_{2}$ & -0.4 & 10.52 & 4.68 & 20 & 2175 \\
$\mathrm{H}$ & -0.3 & 11.11 & 5.99 & 21.0 & 2278 \\
$X^{2}$ & -0.9 & 6.71 & 4.14 & 19.0 & 2000 \\
EMD & $-\mathbf{0 . 1}$ & $\mathbf{5 . 5 3}$ & $\mathbf{3 . 3}$ & $\mathbf{1 4 . 0}$ & $\mathbf{1 8 6 7}$ \\
\hline
\end{tabular}

\begin{tabular}{|c|c|c|c|c|c|}
\hline \multicolumn{6}{|c|}{ Blocksize 32x32 } \\
\hline$H_{d}$ & $V_{f}$ & Mean & StDev & $\mathrm{R}$ & {$[\mathrm{ms}]$} \\
\hline \hline \multicolumn{6}{|c|}{ non-overlapping } \\
\hline$L_{1}$ & 1.3 & 10.05 & 3.46 & 18.0 & 258 \\
$L_{2}$ & 1.7 & 11.13 & 3.87 & 17.0 & 261 \\
$\mathrm{H}$ & 1.0 & 9.45 & 3.81 & 18.0 & 267 \\
$X^{2}$ & 0.6 & 10.88 & 4.11 & 19.0 & 260 \\
EMD & $\mathbf{1 . 2}$ & $\mathbf{9 . 3 4}$ & $\mathbf{3 . 4 9}$ & $\mathbf{1 6 . 0}$ & $\mathbf{2 6 2}$ \\
\hline \hline \multicolumn{6}{|c|}{ half-overlapping } \\
\hline$L_{1}$ & -0.3 & 8.03 & 3.03 & 14.0 & 1103 \\
$L_{2}$ & -0.2 & 8.44 & 3.12 & 15.0 & 1090 \\
$\mathbf{H}$ & $-\mathbf{0 . 3}$ & $\mathbf{7 . 1 3}$ & $\mathbf{3 . 1 6}$ & $\mathbf{1 4 . 0}$ & $\mathbf{1 0 7 8}$ \\
$X^{2}$ & -0.6 & 8.12 & 3.05 & 15.0 & 1064 \\
EMD & 0.4 & 8.35 & 3.57 & 17.0 & 1043 \\
\hline
\end{tabular}

Table 1. Experiment $\# 1$ - Statistical analyisis

Results show that smaller block sizes and overlapping blocks increase the segmentation accuracy. Especially for shape estimation, a smaller distance between the block-center points leads to a refinement and increases the accuracy of the CS boundary estimation. On the other hand, the amount of blocks is a crucial factor for the timing performance. With an increasing amount of blocks the timing performance decreases significantly. For example, compare the nonand half-overlapping timing measurements in Table 1 .

Furthermore, the results show that for non-overlapping blocks lower variance factors $\left(V_{f}\right)$ and for half-overlapping blocks higher variance factors are required. 
Overlapping and smaller blocks are more similar than larger sized ones which are not overlapping. Thus, the variance factors for larger sized blocks need to be higher and consequently less restrictive. Smaller block sizes and overlapping blocks require lower variance factors and enable a more accurate segmentation.

\subsection{Experiment \#2 - LBP histograms}

Second, LBPs are examined as texture features. For this purpose, equal settings as in Experiment $\# 1$ are utilized. Due to an improper ordering of the histogram bins the EMD is not evaluated. Four different LBP variations (3x3 neighbourhood) are evaluated: basic LBPs, uniform LBPs, multiscale LBPs (three scales - see [29]) and multiscale \& uniform LBPs. In Table 2 the best results for certain block settings and LBP variations are shown.

Nearly all of the most accurate results for a certain configuration are computed using the $L_{2}$ norm. Contrary to the first experiment, smaller blocksizes and over-

\begin{tabular}{|c|c|c|c|c|c|c|}
\hline \multicolumn{7}{|c|}{ LBP histograms } \\
\hline Config. & $H_{d}$ & $V_{f}$ & Mean & StDev & $\mathrm{R}$ & [ms] \\
\hline \multicolumn{7}{|c|}{$3 \times 3$ LBPs } \\
\hline \begin{tabular}{|l|l|}
$16 \times 16$ & no
\end{tabular} & $L_{2}$ & 0.9 & 9.85 & 4.75 & 20.0 & 772 \\
\hline $32 \times 32$ ho & $L_{2}$ & -0.2 & 8.88 & 3.83 & 25.0 & 3273 \\
\hline \multicolumn{7}{|c|}{$3 \times 3$ uniform LBPs } \\
\hline \begin{tabular}{|l|l|}
$16 \times 16$ & ho
\end{tabular} & $X^{2}$ & -0.4 & 8.57 & 2.91 & 14.0 & 6636 \\
\hline $32 \times 32$ no & $L_{2}$ & 0.5 & 9.66 & 3.64 & 14.0 & 711 \\
\hline \multicolumn{7}{|c|}{ 3x3 multiscale LBP } \\
\hline \begin{tabular}{|l|l}
$16 \times 16$ & no \\
\end{tabular} & $L_{2}$ & 0.0 & 9.37 & 7.31 & 73 & 1294 \\
\hline $32 \times 32$ ho & $L_{2}$ & 0.3 & 7.53 & 3.45 & 14.0 & 5084 \\
\hline \multicolumn{7}{|c|}{$3 \times 3$ multiscale \& uniform LBPs } \\
\hline \begin{tabular}{|l|l|}
$16 \times 16$ & no
\end{tabular} & $L_{1}$ & 0.9 & 8.89 & 4.29 & 16.0 & 1250 \\
\hline $32 \times 32$ ho & $L_{2}$ & 0.1 & 7.5 & 3.41 & 15.0 & 5206 \\
\hline
\end{tabular}

Table 2. Experiment \#2 Statistical Analysis (no $=$ non-overlapping, ho = half-overlapping) lapping blocks do not increase the segmentation accuracy. Due to strongly varying accuracies over all results it is not possible to draw further conclusions about the impact of the blocksize and half-/ non-overlapping blocks on the accuracy.

The most accurate result is achieved using the $L_{1}$ norm and multiscale \& uniform LBPs $\left(L_{1} / 32 \times 32 /\right.$ half-overlapping - Mean: 7.53, StDev: 3.41). It is difficult to conclude that using multiscale LBPs or uniform LBPs instead of normal 3x3 LBPs improves the segmentation accuracy. However, the two most accurate results are reached with the LBP extensions and especially with multiscale \& uniform LBPs.

Results show that the two LBP extensions influence the timing performance differently. While for uniform LBPs the number of bins in the LBP histogram decreases, for multiscale LBPs the number of bins increases with each scale. Consequently, uniform LBPs increase the timing performance and multiscale LBPs cause a significant deterioration of the timing performance.

The most important conclusion is that the results of the first experiment are more robust over all configurations and histogram distances. The best results of the first experiment using intensity histograms remarkable outperform the segmentation accuracies and timing measurements of the second experiment using LBP histograms.

\subsection{Experiment \#3 - Circle/ellipse fitting}

To assess the impact of the circle/ellipse fitting step the best configurations (Intensity histograms/ EMD/ 16x16 pixels blocks) are recomputed including 
circle/ellipse fitting (Table 3). Compared to the corresponding results in Table 1 the results in Table 3 show that the circle/ellipse fit step is increasing the segmentation accuracy. Unfortunately, the circle-/ellipse fit approach is very time consuming.

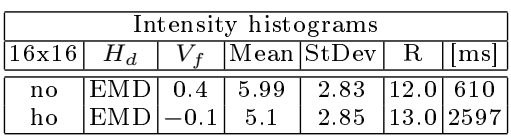

Table 3. Experiment \#3 Circle/ellipse fitting improvement

\subsection{Timing performance evaluation}

For evaluation of the timing performance, the testset images were scaled in a range from $\{0.1,0.2, \ldots, 1, \ldots, 2\}$ according to the amount of pixels. For each scale, the segmentation accuracies and timings are computed as described in Experiment \#1 using EMD and half-overlapping blocks. The most accurate results for each scale and blocksize are used to illustrate the timing performance in Fig. 5

Compared to $32 \times 32$ blocks, with $16 \times 16$ blocks the amount of analysed

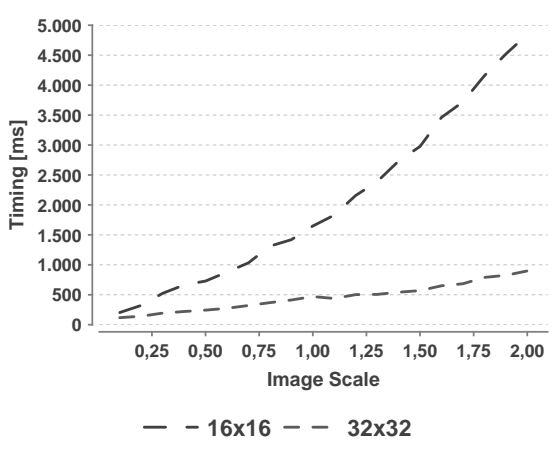

Fig. 5. Timing performance blocks increases approximately by a factor of 4 . Results show, that the timings for $32 \times 32$ blocks increase roughly linearly. The different increase of the $16 \times 16$ timings is caused by the Alpha shape computation step which has a complexity of $\mathrm{O}(\mathrm{n} \log n)$. In case of $16 \times 16$ blocks and larger scales, the number of points used for Alpha shape computation increases rapidly. Thus, the impact on the timing performance becomes clearly visible. Finally, it can be stated that an appropriate selection of the blocksize is crucial for the timing performance and the accuracy of the CS segmentation algorithm.

\section{Conclusion}

We proposed a similarity-based region growing algorithm which is suitable for segmentation of CS in images of rough log end faces. In combination with intensity histograms as texture features and the EMD as histogram distance our algorithm is fast, accurate and robust.

Surprisingly, LBP histograms as texture features achieve less accurate and very irregular results. Additionally, the timing performance decreases remarkable. These observations lead to an interesting conclusion. For particular segmentation applications, simple texture features and a more sophisticated similarity measure can outperform matured texture features and a simple similarity measure.

The experimental evaluation of our work forms a solid basis for further research on CS segmentation. In our future research further segmentation techniques will be evaluated based on their accuracy and timing performance. Apart 
from the computational effort, active contour approaches as presented by [27] are of interest because they are probably more robust to similar textured crosssections in the background.

\section{References}

1. Wu, J., Liew, D.: A computer vision method for detection of external log cracks and pith in log cross-section images. In: Procs. of the World Automation Congress: International Symposium on Intelligent Automation and Control (ISIAC'00), Hawaii, USA (2000)

2. Hanning, T., Kickingereder, R., Casasent, D.: Determining the average annual ring width on the front side of lumber. In Osten, W., Kujawinska, M., Creath, K., eds.: Proceedings of SPIE: Optical Measurement Systems for Industrial Inspection. Volume 5144., Munich, Germany (2003) 707-716

3. Österberg, P., Ihalainen, H., Ritala, R.: Method for analyzing and classifying wood quality through local 2d-spectrum of digital log end images. In: Proceedings of International Conference on Advanced Optical Diagnostics in Fluids, Tokyo, JP (2004)

4. Norell, K., Borgefors, G.: Estimation of pith position in untreated log ends in sawmill environments. Computers and Electronics in Agriculture 63 (2008) 155167

5. Schraml, R., Uhl, A.: Pith estimation on rough log end images using local fourier spectrum analysis. In: Proceedings of the 14th Conference on Computer Graphics and Imaging (CGIM'13), Innsbruck, AUT (2013)

6. Cerda, M., Hitschfeld-Kahler, N., Mery, D.: Robust tree-ring detection. In Mery, D., Rueda, L., eds.: Procs. of Advances in Image and Video Technology. Volume 4872 of LNCS., Springer Berlin / Heidelberg (2007) 575-585

7. Norell, K.: An automatic method for counting annual rings in noisy sawmill images. In: Proceedings of the Conference on Image Analysis and Processing (ICIAP). Number 5716 in LNCS, Springer Berlin / Heidelberg (2009) 307-316

8. Norell, K.: Counting annual rings on pinus sylvestris end faces in sawmill industry. Computers and Electronics in Agriculture 75 (2010) 231-237

9. Longuetaud, F., Mothe, F., Kerautret, B., Krähenbühl, A., Hory, L., Leban, J.M., Debled-Rennesson, I.: Automatic knot detection and measurements from x-ray ct images of wood: A review and validation of an improved algorithm on softwood samples. Computers and Electronics in Agriculture 85 (2012) 77-89

10. Schraml, R., Uhl, A.: Temporal and longitudinal variances in wood log crosssection image analysis. In: IEEE International Conference on Image Processing 2014 (ICIP 2014), Paris, FR (2014)

11. Boykov, Y., Jolly, M.P.: Interactive graph cuts for optimal boundary \& region segmentation of objects in n-d images. In: Proceedings of the 8th IEEE International Conference on Computer Vision (ICCV'01). Volume 1. (2001) 105-112

12. Boykov, Y., Funka-Lea, G.: Graph cuts and efficient n-d image segmentation. International Journal of Computer Vision 70 (2006) 109-131

13. Chan, T., Vese, L.: Active contours without edges. IEEE Transactions on Image Processing 10 (2001) 266-277

14. Chan, T., Esedoglu, S., Ni, K.: Histogram based segmentation using wasserstein distances. In: Proceedings of the 1st international conference on Scale space and variational methods in computer vision. SSVM'07, Ischia, ITA, Springer Berlin / Heidelberg (2007) 697-708 
15. Comaniciu, D., Meer, P.: Mean shift: a robust approach toward feature space analysis. IEEE Transactions on Pattern Analysis and Machine Intelligence 24 (2002) 603-619

16. Haindl, M., Mikeš, S.: Unsupervised texture segmentation using multiple segmenters strategy. In: Proceedings of the 7th International Conference on Multiple Classifier Systems (MCS'07), Berlin, Heidelberg, Springer-Verlag (2007) 210-219

17. Unser, M.: Texture classification and segmentation using wavelet frames. IEEE Transactions on Image Processing 4 (1995) 1549-1600

18. Wang, B., Zhang, L.: Supervised texture segmentation using wavelet transform. In: Proceedings of the International Conference on Neural Networks and Signal Processing. Volume 2. (2003) 1078-1082

19. Weldon, T.P., Higgins, W.E., Dunn, D.F.: Efficient gabor filter design for texture segmentation. Pattern Recognition 29 (1996) 2005 - 2015

20. Eiterer, L., Facon, J., Menoti, D.: Postal envelope address block location by fractalbased approach. In: Proceedings of the 17th Brazilian Symposium on Computer Graphics and Image Processing. (2004) 90-97

21. Materka, A., Strzelecki, M.: Texture analysis methods - a review. Technical report, Institute of Electronics, Technical University of Lodz (1998)

22. de Oliveira Nunes, E., Conci, A.: Texture segmentation considering multiband, multiresolution and affine invariant roughness. In: XVI Brazilian Symposium on Computer Graphics and Image Processing. (2003) 254-261

23. Stitou, Y., Turcu, F., Berthoumieu, Y., Najim, M.: Three-dimensional textured image blocks model based on wold decomposition. Signal Processing, IEEE Transactions on 55 (2007) 3247-3261

24. Eckley, I.A., Nason, G.P., Treloar, R.L.: Locally stationary wavelet fields with application to the modelling and analysis of image texture. Journal of the Royal Statistical Society: Series C (Applied Statistics) 59 (2010) 595-616

25. Atto, A., Berthoumieu, Y., Bolon, P.: 2-d wavelet packet spectrum for texture analysis. Image Processing, IEEE Transactions on 22 (2013) 2495-2500

26. Jung, M., Peyré, G., Cohen, L.D.: Texture segmentation via non-local nonparametric active contours. In: Proceedings of the 8th International Conference on Energy Minimization Methods in Computer Vision and Pattern Recognition (EMMCVPR'11), Berlin, Heidelberg, Springer-Verlag (2011) 74-88

27. Jung, M., Peyré, G., Cohen, L.D.: Nonlocal active contours. SIAM Journal on Imaging Sciences 5 (2012) 1022-1054

28. Ojala, T., Pietikäinen, M., Harwood, D.: A comparative study of texture measures with classification based on featured distributions. Pattern Recognition 29 (1996) $51-59$

29. Ojala, T., Pietikainen, M., Maenpaa, T.: Multiresolution gray-scale and rotation invariant texture classification with local binary patterns. IEEE Transactions on Pattern Analysis and Machine Intelligence 24 (2002) 971-987

30. Mäenpää, T.: The Local Binary Pattern Approach to Texture Analysis - Extensions and Applications. PhD thesis, University of Oulu (2003)

31. Rubner, Y., Tomasi, C., Guibas, L.: A metric for distributions with applications to image databases. In: Computer Vision, 1998. Sixth International Conference on. (1998) 59-66

32. Edelsbrunner, H., Kirkpatrick, D., Seidel, R.: On the shape of a set of points in the plane. Information Theory, IEEE Transactions on 29 (1983) 551-559 